\title{
Little Exercise, Big Effects: Reversing Aging and Infection-Induced Memory Deficits, and Underlying Processes
}

\author{
Ruth M. Barrientos, Matthew G. Frank, Nicole Y. Crysdale, Timothy R. Chapman, Jared T. Ahrendsen, Heidi E.W. Day, \\ Serge Campeau, Linda R. Watkins, Susan L. Patterson, and Steven F. Maier \\ Department of Psychology and Neuroscience, University of Colorado at Boulder, Boulder, Colorado 80309
}

We have previously found that healthy aged rats are more likely to suffer profound memory impairments following a severe bacterial infection than are younger adult rats. Such a peripheral challenge is capable of producing a neuroinflammatory response, and in the aged brain this response is exaggerated and prolonged. Normal aging primes, or sensitizes, microglia, and this appears to be the source of this amplified inflammatory response. Among the outcomes of this exaggerated neuroinflammatory response are impairments in synaptic plasticity and reductions of brain-derived neurotrophic factor (BDNF), both of which have been associated with cognitive impairments. Since it has been shown that physical exercise increases BDNF mRNA in the hippocampus, the present study examined voluntary exercise in 24-month-old F344 $\times \mathrm{BN}$ rats as a neuroprotective therapeutic in our bacterial infection model. Although aged rats ran only an average of $0.7 \mathrm{~km}$ per week, this small amount of exercise was sufficient to completely reverse infection-induced impairments in hippocampusdependent long-term memory compared with sedentary animals. Strikingly, exercise prevented the infection-induced exaggerated neuroinflammatory response and the blunted BDNF mRNA induction seen in the hippocampus of sedentary rats. Moreover, voluntary exercise abrogated age-related microglial sensitization, suggesting a possible mechanism for exercise-induced neuroprotection in aging.

\section{Introduction}

One of the more important advances in recent neuroscience research is the understanding that there is extensive communication between the immune system and the CNS. Multiple pathways supporting this communication have been described, and as a result of this communication, neural activity is altered quite extensively during a peripheral infection (Maier and Watkins, 1998). Proinflammatory cytokines (e.g., interleukin-1 $\beta$; IL$1 \beta$ ) play a key role in this communication, as they are regulators of host responses to infection, inflammation, and reactions to stress or trauma. Importantly, this immune-to-brain signaling results in de novo production of proinflammatory cytokines within the brain, primarily by microglia (Van Dam et al., 1995).

Aged rats show a particular vulnerability to hippocampaldependent long-term memory impairments following a peripheral Escherichia coli (E. coli) infection, which is paralleled by an exaggerated and long-lasting proinflammatory cytokine response in the hippocampus, effects not observed in young adult rats (Barrientos et al., 2006, 2009). Blockade of IL- $1 \beta$ signaling in

Received May 5, 2011; revised June 7, 2011; accepted June 23, 2011.

Author contributions: R.M.B., L.W., and S.F.M. designed research; R.M.B., M.G.F., N.Y.C., T.R.C., J.T.A., H.D., and S.L.P. performed research; H.D., S.C., and S.L.P. contributed unpublished reagents/analytic tools; R.M.B. and M.G.F. analyzed data; R.M.B. and S.F.M. wrote the paper.

This work was supported by National Institute on Aging Grants R01AG02827 (R.M.B., M.G.F., L.R.W., S.F.M.) and R21AG031467 (S.L.P.). We thank Jennifer Hoover and Giuseppe P. Cortese for their skillful technical assistance.

Correspondence should be addressed to Dr. Ruth M. Barrientos, Department of Psychology and Neuroscience, Campus Box 345, University of Colorado at Boulder, Boulder, C0 80309-0345. E-mail: ruth.barrientos@colorado.edu. DOI:10.1523/JNEUROSCI.2266-11.2011

Copyright $\odot 2011$ the authors $\quad 0270-6474 / 11 / 3111578-09 \$ 15.00 / 0$ the brain, with administration of IL-1 receptor antagonist (IL1RA), eliminates the memory impairment (Frank et al., 2010b), underscoring the prominent role of IL- $1 \beta$ in the underlying neural processes. The potentiated proinflammatory cytokine response in hippocampus of aged rats has been shown to be due, at least in part, to a priming of microglial cells that occurs with normal aging (Godbout et al., 2005; Frank et al., 2006, 2010a). Morphological and antigenic markers of activation (e.g., MHCII) are upregulated in hippocampal microglia of aged rats compared with those of young adults (Frank et al., 2006), and the neuroinflammatory response to a proinflammatory stimulus is potentiated (Godbout et al., 2005; Frank et al., 2010a).

The exaggerated neuroinflammatory response following a peripheral infection in aged rats produces a dramatic and specific deficit in a form of long-lasting synaptic plasticity that is dependent on brain-derived neurotrophic factor (BDNF) (Chapman et al., 2010). BDNF is a neuronal growth factor that is rapidly and specifically expressed in the hippocampus during contextual learning (Hall et al., 2000), and is crucial for the formation of long-term memories that depend on the hippocampus (Tyler et al., 2002; Soule et al., 2006); and again, blocking IL-1 $\beta$ signaling with IL-1RA protected against failure of long-lasting synaptic plasticity (Chapman et al., 2010), and BDNF reductions (Cortese et al., 2011) in aged rats following infection.

Here, we examined voluntary exercise in aged rats as a possible therapeutic intervention to ameliorate the cascade of maladaptive responses that follow peripheral infection, including (1) memory impairments, (2) reduced BDNF induction, (3) potentiated neuroinflammation, and (4) microglial sensitization. In 
young subjects, physical exercise has been shown to increase hippocampal BDNF (Cotman et al., 2007), enhance memory performance on tasks that depend on the hippocampus via upregulation of BDNF (Vaynman et al., 2004), and ameliorate the neuroinflammatory response following an immune challenge (Nickerson et al., 2005). However, whether exercise would reduce aging-induced susceptibility to the cognitive impairments that follow peripheral infection, and the processes thought to underlie these impairments, has not been explored.

\section{Materials and Methods}

Subjects. Subjects were male F $344 \times$ BN F1 rats obtained from the National Institute on Aging (Bethesda, MD). Upon arrival at our facility, aged rats were 22 months old, weighed $\sim 575 \mathrm{~g}$, and were housed singly in Plexiglas cages $(52 \mathrm{~L} \times 30 \mathrm{~W} \times 21 \mathrm{H}, \mathrm{cm})$. All rats were allowed ad libitum access to food and water and were given 1-2 weeks to acclimate to colony conditions before experimentation began. Runners were housed in a cage with a running wheel (circumference $1.08 \mathrm{~m}$ ). A bicycle computer was attached to each cage/wheel to monitor running. Another group was housed in a cage with a running wheel that was locked in place (Locked) to control for having a novel item in their cage, but these rats were unable to run. A third group did not have a running wheel in their cage (Sedentary). Rats were maintained in these conditions for 6 weeks. The animal colony was maintained at $22 \pm 1^{\circ} \mathrm{C}$ on a $12 \mathrm{~h}$ light/dark cycle (lights on at 7:00 A.M.). All experiments were conducted in accordance with protocols approved by the University of Colorado Animal Care and Use Committee. All efforts were made to minimize the number of animals used and their suffering.

Immune challenge. In all but the glial sensitization experiments, animals received an intraperitoneal injection of either Escherichia coli (E. coli) (a ubiquitous bacterial strain), or vehicle. One day before administration, stock cultures were thawed and cultured overnight $(15-20 \mathrm{~h})$ in $40 \mathrm{ml}$ of brain-heart infusion (DIFCO) in an incubator $\left(37^{\circ} \mathrm{C}, 95 \%\right.$ air + $5 \% \mathrm{CO}_{2}$ ). The number of bacteria in cultures was quantified by extrapolating from previously determined growth curves. Cultures were then centrifuged for $15 \mathrm{~min}$ at $4^{\circ} \mathrm{C}, 3000 \mathrm{rpm}$, supernatants discarded, and bacteria resuspended in sterile PBS. Bacteria were resuspended with a volume of PBS to achieve a dose of $1.0 \times 10^{10}$ colony-forming units. A volume of $250 \mu \mathrm{l}$ was injected intraperitoneally. Vehicle-treated rats received an injection of sterile PBS of an equal volume $(250 \mu \mathrm{l})$.

Contextual fear conditioning. We chose to use the immediate-shock fear conditioning paradigm (Fanselow, 1990; Rudy et al., 2002) as the learning task. Here, foot shock is delivered very quickly upon exposure to the experimental context on the fear conditioning day. However, the rats are preexposed to the context at an earlier time. The preexposures are required so that the subjects are able to form a "conjunctive representation" of the context, so that it can be associated with the shock on the conditioning day (Rudy et al., 2002). We chose this fear conditioning paradigm because it is highly and specifically dependent on the hippocampus (Rudy et al., 2002), and we have already shown that E. coli interferes with memory of this task in aging rats (Barrientos et al., 2006). The conditioning context was one of two identical Igloo ice chests (54 $\mathrm{L} \times 30 \mathrm{~W} \times 27 \mathrm{H}, \mathrm{cm}$ ) with white interiors. A speaker and an activated 24 V DC light bulb were mounted on the ceiling of each chest. The conditioning chambers $(26 \mathrm{~L} \times 21 \mathrm{~W} \times 24 \mathrm{H}, \mathrm{cm})$, placed inside each chest, were made of clear plastic and had window screen tops. A foot shock was delivered through a removable floor of stainless steel rods $1.5 \mathrm{~mm}$ in diameter, spaced $1.2 \mathrm{~cm}$ center to center. Each rod was wired to a shock generator and scrambler (Colbourn Instruments). Chambers were cleaned with water before each animal was conditioned or tested.

Rats were taken two at a time from their home cage and transported to the conditioning context in a black bucket with the lid on so that the rats could not see where they were being taken. This procedure was used to establish an association between the contextual representation of the conditioning context and the transport cues that precede placement of the rat in the context. Rats were placed in the context and allowed to freely explore and then were transported back to their home cage, where they remained $\sim 40 \mathrm{~s}$ before the next exposure. This procedure was re- peated 3 times. Animals remained in the novel context for $5 \mathrm{~min}$ on the first exposure and for $40 \mathrm{~s}$ on two subsequent exposures. The rats were transported in the black bucket each time that they were returned to their home cage, but with the lid off. The purpose of these multiple exposures was to establish the features of the black bucket as retrieval cues that could activate the representation of the context (for further details, see Rudy et al., 2002). Seventy-two hours later, each animal was taken from its home cage and transported to the conditioning context in the black bucket. There, they received one $2 \mathrm{~s}, 1.5 \mathrm{~mA}$ shock immediately after being placed in the context. They were then quickly taken out of the context and transported back to their home cage. The rats' time in the conditioning context on this day never exceeded $10 \mathrm{~s}$. Fear of the conditioning context was assessed $24 \mathrm{~h}$ after immediate shock ( $4 \mathrm{~d}$ after preexposure) by placing the rat in the conditioning context for 6 min. Several hours later, generalized fear was assessed by placing the rat in an alternate context for $6 \mathrm{~min}$ also. Every $10 \mathrm{~s}$ each rat was judged as either freezing or active at the instant the sample was taken. Freezing, the rat's dominant defensive fear response, is a complete suppression of behavior that is accompanied by immobility, shallow breathing, and a variety of other autonomic changes including an increase in heart rate and piloerection (Fanselow and Lester, 1988). Freezing in these experiments was defined as the absence of all visible movement, except for respiration. Scoring began $\sim 10 \mathrm{~s}$ after the animal was placed into the chamber. Scoring was performed by observers blind to experimental treatment, and inter-rater reliability exceeded $97 \%$ for all experiments.

Tissue dissection. For in situ hybridization experiments, animals were rapidly decapitated one at a time $2 \mathrm{~h}$ postconditioning. Age-matched home cage control rats were also killed in this manner. Brains were removed, rapidly frozen in isopentane, and stored at $-70^{\circ} \mathrm{C}$. For all other experiments, animals were given a lethal dose of sodium pentobarbital and transcardially perfused with ice-cold saline $(0.9 \%)$ for $3 \mathrm{~min}$ to remove peripheral immune leukocytes from the CNS vasculature. Brains were then rapidly extracted and placed on an ice-cold frosted glass plate, and hippocampi were dissected for both IL- $1 \beta$ protein assay and microglia cell isolations. Liver was also dissected for measurement of IL- $1 \beta$ protein. Tissues slated for protein measurements were quickly frozen in liquid nitrogen and stored at $-70^{\circ} \mathrm{C}$ until the time of processing. For methods on how tissues were processed for microglial isolation experiments, see below.

In situ hybridization and histology. Brains were cut (10 $\mu \mathrm{m}$ slices) on a Leica cryostat through the dorsal hippocampus, thaw-mounted onto poly-L-lysine-coated slides, and stored at $-70^{\circ} \mathrm{C}$ until processing. Sections were fixed in buffered paraformaldehyde (4\%) for $1 \mathrm{~h}$, rinsed three times with $2 \times$ SSC (standard sodium citrate), placed in $0.1 \mathrm{M}$ triethanolamine containing $0.25 \%$ acetic anhydride for $10 \mathrm{~min}$, rinsed for $5 \mathrm{~min}$ in $\mathrm{H}_{2} \mathrm{O}$, and dehydrated in alcohols. The cDNA probe specific for BDNF generated by standard reverse transcriptase (RT)-PCR and subcloned into pSC-A plasmid (Stratagene) was directed at exon IX, which codes for the mature protein (GenBank accession number EF125675.1, nt 6611410). It was linearized using the NheI restriction enzyme. To generate ${ }^{35}$ S-labeled complementary RNA to BDNF mRNA, $1 \mu \mathrm{g}$ of linearized plasmid DNA, $5 \mu \mathrm{l}$ of $5 \times$ transcription buffer (Promega), $7.5 \mu \mathrm{l}$ of ${ }^{35} \mathrm{~S}$-UTP, $4 \mu \mathrm{l}$ of $\mathrm{H}_{2} \mathrm{O}, 2.5 \mu \mathrm{l}$ of $0.1 \mathrm{M}$ dithiothreitol (DTT), $1 \mu \mathrm{l}$ each of $100 \mathrm{~mm}$ GTP, CTP, and ATP, $1 \mu \mathrm{l}$ of RNase inhibitor, and $1 \mu \mathrm{l}$ of T3 polymerase in a total volume of $25 \mu \mathrm{l}$ were incubated for $2 \mathrm{~h}$ at $37^{\circ} \mathrm{C}$. To isolate the complete complementary RNA from single nucleotides, a Sephadex G50-50 column was used. The ${ }^{35}$ S-labeled probe was diluted in hybridization buffer to yield an approximate concentration of $1 \times 10^{6}$ $\mathrm{cpm} / 65 \mu \mathrm{l}$. The hybridization buffer consisted of 50\% formamide, $10 \%$ dextran sulfate, $2 \times$ SSC, 50 mm sodium phosphate buffer, $\mathrm{pH} 7.4,1 \times$ Denhardt's solution, and $0.1 \mathrm{mg} / \mathrm{ml}$ yeast tRNA. The radiolabeled probe/ hybridization mixture $(65 \mu \mathrm{l})$ was applied to each slide, and sections were coverslipped. Slides were placed in covered plastic boxes lined with filter paper moistened with $50 \%$ formamide $/ 50 \% \mathrm{H}_{2} \mathrm{O}$ and incubated for $12-16 \mathrm{~h}$ at $55^{\circ} \mathrm{C}$. Coverslips were floated off in $2 \times$ SSC, and slides were rinsed three times in $2 \times$ SSC. Slides were incubated in RNase A (200 $\mu \mathrm{g} / \mathrm{ml}$ ) for $60 \mathrm{~min}$ at $37^{\circ} \mathrm{C}$, followed by successive washes in $2 \times, 1 \times$, $0.5 \times$, and $0.1 \times$ SSC for $2-3 \mathrm{~min}$ each, with an additional incubation in $0.1 \times$ SSC for $60 \mathrm{~min}$ at $70^{\circ} \mathrm{C}$. Slides were rinsed in distilled $\mathrm{H}_{2} \mathrm{O}$, 
dehydrated in alcohols, and exposed to Kodak BIOMAX MR x-ray film for $7 \mathrm{~d}$.

Semiquantitative analyses were performed on digitized images from $\mathrm{x}$-ray films in the linear range of the acquisition system. This system consisted of a Northern Light box, model B-95 (Imaging Research Inc.), a Sony XC-ST70 digital camera fitted with a Navitar 7000 zoom lens, a Scion Corp. LG3 frame grabber board, and Scion Image (ver. Beta 4.0.2) on a Dell 8100 computer. Signal pixels of a region of interest were defined as having a gray value of $3.5 \mathrm{SDs}$ above the mean gray value of a cell-poor area close to the region of interest. The number of pixels and the average gray values above the set background were then computed for the region of interest and multiplied, giving an integrated densitometric measurement. Based on our recent electrophysiology findings showing a substantial deficit in long-lasting synaptic plasticity in CA1 neurons of infected aged rats (Chapman et al., 2010), we chose to focus on the CA1 region of the hippocampus here. An average of 8 to 12 measurements were made for this region, and these values were further averaged to obtain a single integrated density value for each rat. The specificity of the probe was confirmed in a control experiment by using a sense probe. No specific hybridization was observed in the sense-treated sections (data not shown).

Detection of IL-1 $\beta$ protein. To prepare the tissue for the assay, each tissue was added to $0.25-0.5 \mathrm{ml}$ of Iscove's culture medium containing $5 \%$ fetal calf serum and an enzyme inhibitor mixture (containing, in mм: 100 amino- $n$-caproic acid, 10 EDTA, 5 benzamidine $\mathrm{HCl}$, and 0.2 phenylmethylsulfonyl fluoride). Total protein was mechanically dissociated from tissue using a sonic dismembrator (Branson Ultrasonics Corp, Model 150E). Sonication consisted of $20 \mathrm{~s}$ of cell disruption at $52 \%$ amplitude. Sonicated samples were centrifuged at $10,000 \times g$ at $4^{\circ} \mathrm{C}$ for $10 \mathrm{~min}$. Supernatants were removed and stored at $4^{\circ} \mathrm{C}$ until ELISA was performed. Bradford protein assays were also performed to determine total protein concentrations in sonication samples.

Levels of IL- $1 \beta$ protein were determined using a commercially available rat IL- $1 \beta$ ELISA kit (R\&D Systems). The assay was performed according to the manufacturer's instructions. The sensitivity of the assay is $<5 \mathrm{pg} / \mathrm{ml}$. Inter- and intra-assay coefficient of variation is $5.5 \%$ and $4.7 \%$, respectively. A total of $50 \mu \mathrm{l}$ of tissue sonicates were used for the assay, and samples were run in duplicate. The concentration of IL-1 $\beta$ protein is presented as picograms of IL- $1 \beta$ per $100 \mu \mathrm{g}$ of total protein.

Ex vivo immune stimulation of hippocampal microglia with lipopolysaccharide. Twenty-four-month-old subjects that had 6 weeks prior access to either a running wheel or a locked wheel were given a lethal dose of sodium pentobarbital and transcardially perfused with ice-cold saline $(0.9 \%)$ as described above. Brains were then rapidly extracted and placed on an ice-cold frosted glass plate, and hippocampi were dissected. Hippocampal microglia were isolated using a Percoll density gradient as previously described (Frank et al., 2006). Immunophenotype and purity of microglia were assessed using real time RT-PCR. Microglia were suspended in DMEM $+10 \%$ FBS and microglia concentration was determined by trypan blue exclusion. Microglia concentration was adjusted to a density of $5 \times 10^{3}$ cells $/ 100 \mu \mathrm{l}$, and $100 \mu \mathrm{l}$ were added to individual wells of a 96-well v-bottom plate. Lipopolysaccharide (LPS) was then used to challenge microglia in vitro. We have previously determined the optimal in vitro conditions under which LPS stimulates a proinflammatory cytokine response in these cells (Frank et al., 2006). Cells were incubated with 4 different doses of LPS $(0.1,1,10$, and $100 \mathrm{ng} / \mathrm{ml})$ or media alone for $2 \mathrm{~h}$ at $37^{\circ} \mathrm{C}, 5 \% \mathrm{CO}_{2}$. At the end of the incubation, the plate was centrifuged at $1000 \times \mathrm{g}$ for $10 \mathrm{~min}$ at $4^{\circ} \mathrm{C}$ to pellet cells, and cells were washed once in ice-cold PBS and centrifuged at $1000 \times g$ for $10 \mathrm{~min}$ at $4^{\circ} \mathrm{C}$. Cell lysis/homogenization, DNase treatment, and cDNA synthesis were performed using the SuperScript III CellsDirect cDNA Synthesis System (Invitrogen).

Real time RT-PCR measurement of gene expression. A detailed description of the PCR amplification protocol has been published previously (Frank et al., 2006). cDNA sequences were obtained from GenBank at the National Center for Biotechnology Information (NCBI; www.ncbi.nlm.nih.gov). Primer sequences were designed using the Eurofins MWG Operon Oligo Analysis \& Plotting Tool (http://www.operon.com/technical/toolkit.aspx) and tested for sequence specificity using the Basic Local Alignment
Search Tool at NCBI (Altschul et al., 1997). Primers were obtained from Invitrogen. Primer specificity was verified by melt curve analysis. All primers were designed to exclude amplification of genomic DNA. Primer sequences are as follows: $\beta$-actin, forward (F), TTCCTTCCTGGGTATGGAAT, reverse (R), GAGGAGCAATGATCTTGATC; IL- $1 \beta$, F, CCTTGTGCAAGTGTCTGAAG, R, GGGCTTGGAAGCAATCCTTA; IL-6, F, AGAAAAGAGTTGTGCAATGGCA, R, GGCAAATTTCCT GGTTATATCC; TNF $\alpha$, F-CAAGGAGGAGAAGTTCCCA, R,TT GGTGGTTTGCTACGACG.

PCR amplification of cDNA was performed using the Quantitect SYBR Green PCR Kit (Qiagen). Formation of PCR product was monitored in real time using the MyiQ Single-Color Real-Time PCR Detection System (Bio-Rad). Relative gene expression was determined by taking the expression ratio of the gene of interest to $\beta$-actin.

\section{Results}

\section{Distance run and body weight changes}

Aged (22.5-month-old) rats were housed with a running wheel, a locked running wheel, or no wheel. The last two groups served as sedentary controls, while rats provided with a functional wheel were given free, around-the-clock access to the wheels for a period of 6 weeks. Animals given access to a functional running wheel ran an average of $1.0 \mathrm{~km}$ during the first week, and steadily decreased distances until stabilizing at $\sim 0.5 \mathrm{~km}$ the last 2 weeks of the study (Fig. 1a). This is only a small amount of running. In fact, data from our laboratory show that young ( 3 months) rats of the same strain as used here $(\mathrm{F} 344 \times \mathrm{BN})$ run an average of $30 \mathrm{~km}$ per week over a 6 week period (data not shown). Strikingly, aged rats ran nearly 50 times less than young animals. There were no differences in body weight between the three groups at the start of the study (Fig. 1b, inset). However, despite the small amounts of exercise displayed by the aged runners, it is notable that they showed marked weight loss by the first week, compared with both sedentary rats and rats housed with a locked wheel, and maintained that weight loss for the duration of the experiment (Fig. $1 b)$. Interestingly, rats housed with a locked wheel trended toward weight loss also, and indeed had lost significantly more weight compared with sedentary rats on weeks 4,5 and 6 , but remained significantly heavier compared with running rats.

\section{Effects of voluntary wheel-running on $E$. coli-induced contextual fear memory}

Here, we examined the influence of voluntary wheel running on peripheral infection-induced hippocampal-dependent memory consolidation deficits. This was only done in aged rats because $E$. coli does not produce a memory deficit in young rats (Barrientos et al., 2006). After 6 weeks of free access to a running wheel, a locked wheel, or no wheel, 24-month-old rats received a peripheral administration of $E$. coli or vehicle. Contextual fear conditioning occurred $4 \mathrm{~d}$ later. Testing for contextual fear memory, by scoring freezing behavior in a 6 min test, occurred $4 \mathrm{~d}$ after conditioning.

The results are presented in Figure 2. As is typical, E. coli produced a long-term memory impairment for conditioning occurring $4 \mathrm{~d}$ later in sedentary rats. It is known that it is memory that is impaired rather than learning, because prior work has shown that short-term memory tested shortly after conditioning is unaffected (Barrientos et al., 2006), indicating that learning occurs normally. Provision of a locked running wheel did not reduce the memory impairment produced by infection, but free access to a running wheel completely eliminated the impairment (Fig. 2a).

To determine specificity of the freezing response, we scored freezing behavior of all subjects in an alternate context. There 

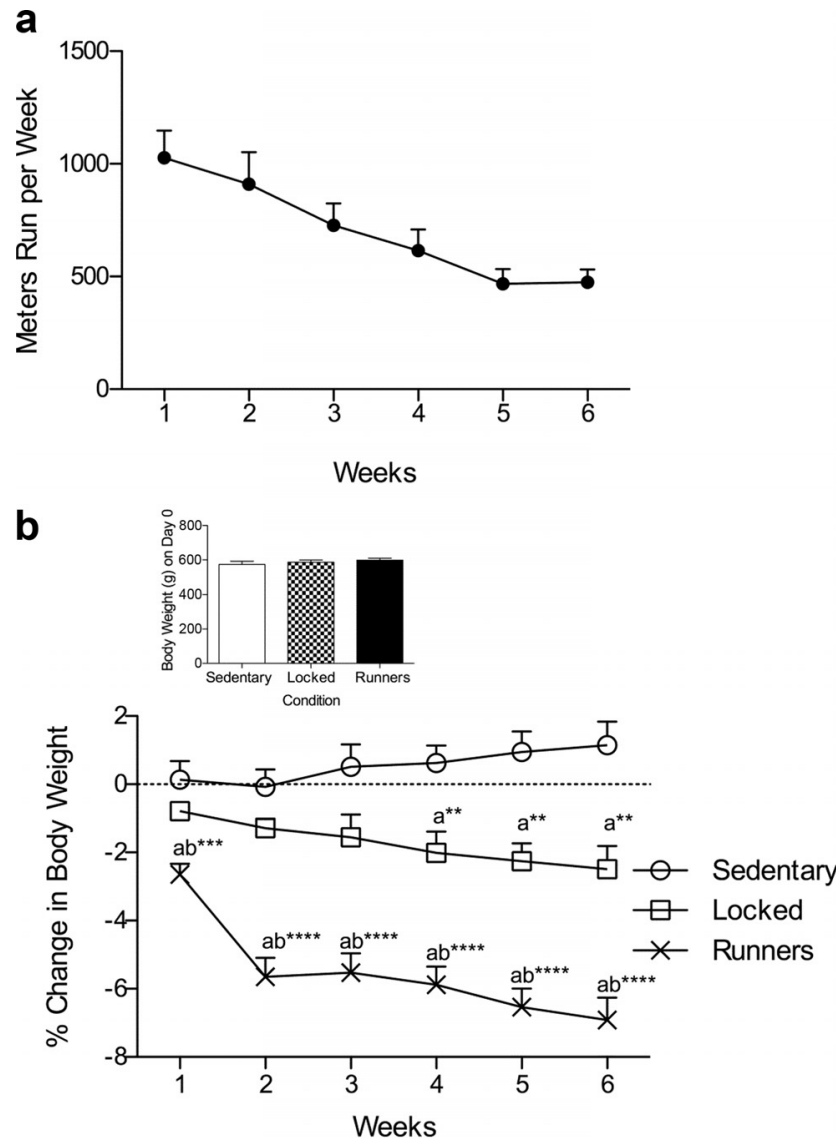

Figure 1. $\boldsymbol{a}, \boldsymbol{b}$, Distance run $(\boldsymbol{a})$, and body weight changes $(\boldsymbol{b})$ over a 6 week period. Means \pm SEM are plotted. $\boldsymbol{a}$, Rats $(n=16)$ ran an average of $1028 \pm 120 \mathrm{~m}$ the first week of the study, and showed a steady decrease over the course of the study with distances stabilizing $\sim 470 \pm 67 \mathrm{~m}$ in weeks 5 and $6 . \boldsymbol{b}$, Inset (starting body weights), A one-way ANOVA showed that there were no differences in body weight at the start of the study among the 3 groups: $F_{(2,31)}=0.87, p>0.05 ; n=8-16$. Percentage body weight changes over 6 weeks, A repeated-measures ANOVA showed there was a significant main effect of physical activity (sedentary, locked, runners), $F_{(2,29)}=42.12, p<0.0001$; a significant main effect of time, $F_{(5,10)}=$ $8.02, p<0.0001$; and a significant physical activity $\times$ time interaction, $F_{(10,145)}=7.90, p<$ 0.0001 . Fisher's post hoc tests revealed that runners $(n=16)$ had lost significantly more weight than did both sedentary rats $(n=8)$ and those with a locked wheel $(n=8)$ beginning with the first week of the study and continuing on through the last $(p<0.0001$, each week). Rats housed with a locked wheel also showed a weight reduction compared with sedentary rats, but these were only significant beginning on week 4 and continuing through week $6(p<0.01$, each week). Sedentary rats gained an average of $0.55 \%$, while rats housed with locked wheels lost an average of $1.73 \%$, and runners lost an average of $5.52 \%$ of their starting weight.

were no differences among the groups (Fig. 2b). These data confirmed that the freezing behavior exhibited in the conditioning context was due to remembering where they had been shocked previously, rather than to generalized fear or anxiety. The fact that freezing in the shock context in the E. coli-treated sedentary rats was no greater than in the non-shock context indicates the magnitude of the memory impairment produced by the $E$. coli infection.

\section{Memory deficits were reversed, not just prevented}

In previous work, rats were tested at 24 months, as here. Since it is unknown whether $E$. coli-induced memory deficits are present at 22.5 months, the age at which exercise was begun, it cannot be concluded whether exercise reversed a deficit that was already present, or prevented the development of the deficit. To answer this question, we determined whether E. coli would impair mem-
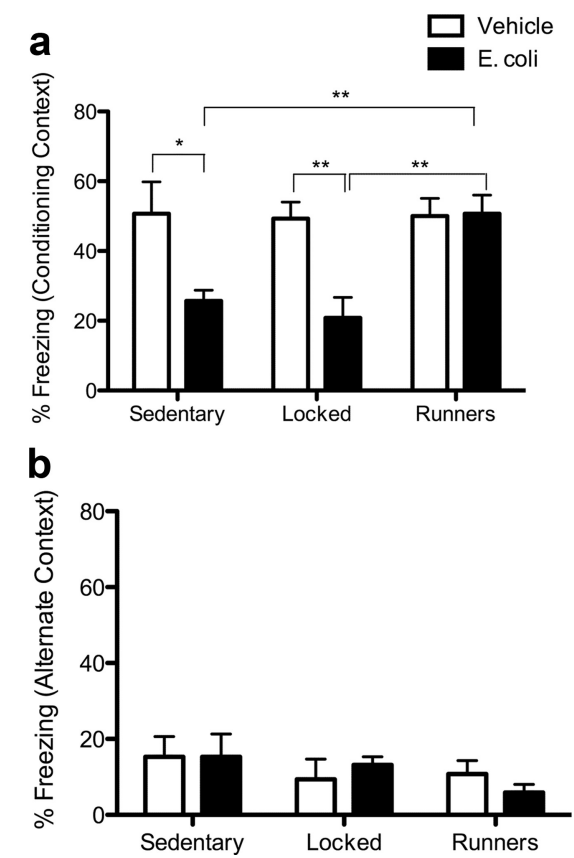

Figure 2. $\quad \boldsymbol{a}, \boldsymbol{b}$, Freezing to the conditioned fear context $(\boldsymbol{a})$ and an alternate context with conditioning having occurred $4 \mathrm{~d}$ after immune challenge $(\boldsymbol{b})$ in 24-month-old rats, following 6 weeks of physical activity. Means \pm SEM are plotted. $\boldsymbol{a}$, There was a significant main effect of physical activity (sedentary, locked, runners), $F_{(2,26)}=4.12, p<0.03$, and a significant main effect of immune challenge (vehicle, $E$. coli), $F_{(1,26)}=11.91, p<0.002$. There was also a significant physical activity $\times$ immune challenge interaction $\left(F_{(2,26)}=4.05, p<0.03\right)$. Fisher's post hoc tests revealed that sedentary $E$. coli-treated rats $(n=4)$ froze significantly less than vehicle-treated controls $(p<0.05 ; n=4)$ and $E$. coli-treated runners $(p<0.01 ; n=8)$. Locked-wheel $E$. coli-treated rats $(n=4)$ also froze significantly less than vehicle controls $(p<$ $0.01 ; n=4)$ and $E$. coli-treated runners $(p<0.01)$. $\boldsymbol{E}$, coli-treated runners showed no difference from their vehicle controls $(p>0.05 ; n=8) . \boldsymbol{b}$, In the alternate context there was not a main effect, either of physical activity, $F_{(2,26)}=1.58, p>0.05$, or of immune challenge $F_{(1,26)}=0.011, p>0.05$.

ory formation at 22.5 months in sedentary rats, thereby indicating that the physiological changes that produce the memory deficit had already taken place. The memory impairment was indeed present. E. coli-treated rats froze significantly less in the conditioning context than did vehicle-treated rats $F_{(1,8)}=5.46$, $p<0.05$. When tested in an alternate context, however, there was no difference between the groups $F_{(1,8)}=2.17, p>0.05$ (graphs not shown), showing that the impairment was specific to the fear memory. These results suggest that voluntary exercise indeed reverses physiological changes that make sedentary aged animals more vulnerable to the memory impairments induced by $E$. coli infection.

\section{Effects of voluntary wheel-running on $E$. coli-induced reductions in BDNF mRNA}

Contextual fear conditioning has been shown to induce BDNF mRNA expression, and this induction is critical to long-term memory formation (Hall et al., 2000; Tyler et al., 2002; Barrientos et al., 2003, 2004; Vaynman et al., 2004; Soule et al., 2006; Cotman et al., 2007; Greenwood et al., 2009). Importantly, elevated levels of proinflammatory cytokines blunt this BDNF increase (Barrientos et al., 2003, 2004). To determine whether voluntary running reduces or prevents the effects of $E$. coli on BDNF expression, we used in situ hybridization to measure BDNF mRNA levels in the CA1 region of the hippocampus $2 \mathrm{~h}$ following contextual fear conditioning. This was done in sedentary, locked 


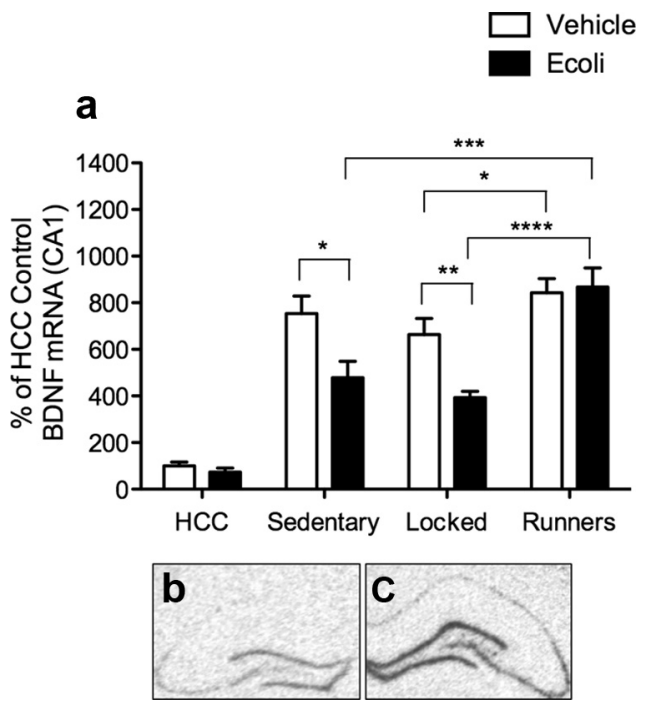

Figure 3. $a, B D N F$ mRNA expression levels in CA1 region of the hippocampus $2 \mathrm{~h}$ after conditioning in rats that received either vehicle or $E$. coli $4 \mathrm{~d}$ before conditioning. Percentages of vehicle $\mathrm{HCC} \pm$ SEM are plotted. There was a significant main effect of physical activity (HCC, sedentary, locked, runners), $F_{(3,43)}=50.144, p<0.0001$, and a significant main effect of immune challenge (vehicle, $E$. coli) $F_{(1,43)}=8.698, p<0.005$. There was also a significant physical activity $\times$ immune challenge interaction $\left(F_{(3,43)}=3.14, p<0.04\right)$. Fisher's post hoc tests showed that all conditioned groups had significantly greater BDNF expression than did vehicle-treated $\mathrm{HCC}$ rats. Vehicle-treated $\mathrm{HCC}(n=5)$ BDNF levels did not differ significantly from those of $E$. coli-treated $\mathrm{HCC}$ rats $(p>0.05 ; n=6)$. Vehicle-treated runners $(n=9)$ exhibited greater BDNF expression compared with vehicle-treated locked wheel rats $(p<0.05$; $n=6)$, but not compared with vehicle-treated sedentary rats $(n=6)$. Sedentary $E$. coli-treated rats $(n=6)$ exhibited significantly blunted BDNF expression compared with their vehicle controls $(p<0.05)$ and $E$. coli-treated runners $(p<0.0005 ; n=8)$. BDNF mRNA levels from locked-wheeled $E$. coli-treated rats $(n=5)$ were also significantly lower compared with their vehicle-treated controls $(p<0.01)$ and $E$. coli-treated runners $(p<0.0001)$. BDNF mRNA levels in $E$. coli-treated runners did not differ from their vehicle-treated controls $(p=0.80) . \boldsymbol{b}, \boldsymbol{c}$, Representative photomicrographs of vehicle-treated HCC (non-conditioned) (b) and conditioned hippocampal (c) slices.

wheel, and running animals that had been administered either vehicle or E. coli $4 \mathrm{~d}$ before being conditioned. Age-matched vehicle- and E. coli-treated home cage control (HCC) rats were also used to assess baseline values. BDNF mRNA levels are presented in Figure 3 as a percentage of the vehicle-treated HCC rats.

All conditioned groups had a robust and significant induction of BDNF mRNA compared with non-conditioned vehicle-treated HCC rats (Fig. 3). BDNF levels of vehicle-treated HCC rats did not differ from that of $E$. coli-treated HCC rats, likely due to a floor effect. Vehicle-treated runners had significantly greater BDNF induction than did vehicle-treated rats housed with a locked wheel, but these values were not significantly greater than those of vehicle-treated sedentary rats. However, E. coli-treated sedentary and locked wheel rats had significantly blunted BDNF mRNA induction compared with their vehicle-treated controls. Strikingly, E. coli-treated runners did not exhibit any blunting, as their BDNF mRNA expression was indistinguishable from vehicle-treated runners, and was significantly greater than all other $E$. coli-treated groups.

These data strongly suggest that voluntary exercise prevents the effects of E. coli on BDNF mRNA induction in the hippocampus of aged rats following a learning experience.

\section{Effects of voluntary wheel-running on E. coli-induced elevations in IL-1 $\beta$}

Aging exaggerates and prolongs the IL- $1 \beta$ increase in the hippocampus that follows the peripheral administration of E. coli
(Barrientos et al., 2006, 2009), and this response has been shown to be responsible for hippocampal-dependent memory deficits, as blocking IL-1 receptors in hippocampus blocks those deficits (Frank et al., 2010b). Given that we have shown that voluntary exercise reverses the E. coli-induced memory impairments, we should expect that this intervention should also reduce hippocampal IL-1 $\beta$ increases after $E$. coli administration in aging subjects. Thus, we measured IL- $1 \beta$ protein $4 \mathrm{~d}$ after $E$. coli in rats that had access to a running wheel, a locked wheel, or no wheel. We measured IL- $1 \beta$ in both the hippocampus and liver. The inflammatory response following an immune challenge in liver has received little attention in aged rats, a significant gap in the literature since inflammatory responses in the liver are especially important in immune-to-brain communication (Campbell et al., 2007).

\section{Hippocampus}

Running had no effect on basal IL-1 $\beta$ protein levels in hippocampus (Fig. $4 a$ ). As is typical, E. coli increased hippocampal IL- $1 \beta$ in sedentary aged rats. The presence of a locked running wheel did not prevent this IL- $1 \beta$ increase. However, rats that had free access to running wheels showed no increase in hippocampal IL- $1 \beta$ at the time point examined. That is, exercise completely blocked the potentiated IL- $1 \beta$ response produced by infection.

\section{Liver}

The pattern in the liver was somewhat different (Fig. 4b). E. coli produced a very large increase in IL- $1 \beta$ that was reduced, but not eliminated by both the locked and the unlocked wheels. The fact that IL- $1 \beta$ levels in liver from E. coli-treated runners were elevated compared with vehicle-treated controls and were not lower than those in E. coli-treated locked wheel rats suggests, not surprisingly, that proinflammatory cytokine levels in the liver are not as critical as hippocampal IL- $1 \beta$ in producing or preventing hippocampal-dependent memory deficits.

\section{Effects of voluntary wheel-running on microglial sensitization}

Sensitization of microglial cytokine responses with age has been suggested as a plausible mechanism facilitating aging-related cognitive vulnerability to infection (Perry, 2004; Frank et al., 2006, 2010a; Henry et al., 2009). Thus, we isolated hippocampal microglia from 24-month-old subjects that had 6 weeks previous access to either a running wheel, or a locked wheel. We did not use a sedentary control to reduce animal usage, and the locked wheel control is the more stringent of the two controls. Hippocampi were dissected and microglia isolated, as previously described (Frank et al., 2010a), and stimulated in vitro with increasing doses of LPS for $2 \mathrm{~h}$. LPS is the major component of the cell walls of Gram-negative bacteria, and is commonly used to stimulate proinflammatory cytokine responses. Gene expression of three proinflammatory cytokines (TNF $\alpha$, IL- $1 \beta$, and IL-6) was measured using real-time PCR.

\section{$T N F \alpha$}

LPS increased TNF $\alpha$ mRNA in a dose-dependent manner, and this increase was potently reduced by prior exercise at all doses of LPS (Fig. 5a).

\section{$I L-1 \beta$}

LPS also increased IL-1 $\beta$ mRNA in a dose-dependent manner, and this increase was also blunted by exercise, in all doses except for the highest dose of LPS (Fig. 5b). 

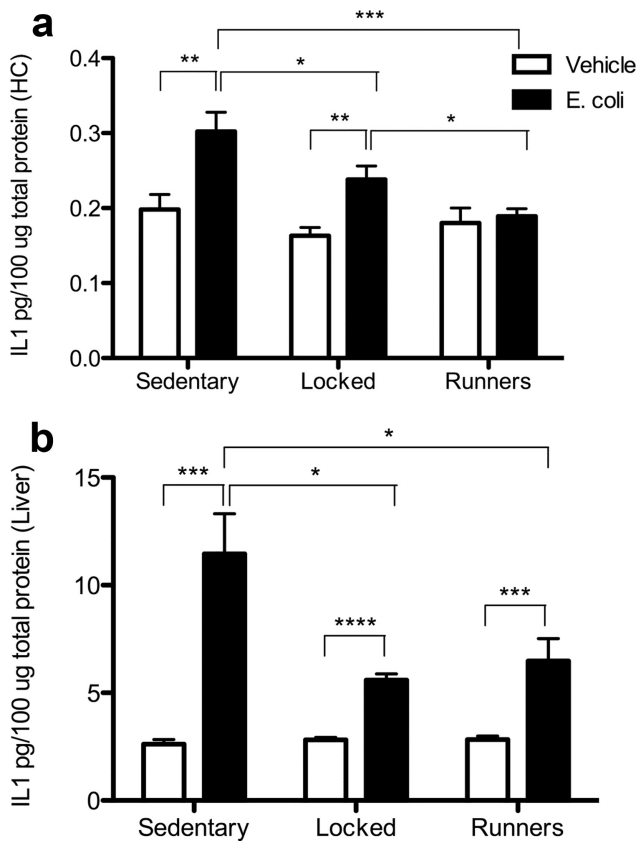

Figure 4. $\boldsymbol{a}, \boldsymbol{b}, \mathrm{IL}-1 \beta$ protein levels in hippocampus $(\boldsymbol{a})$ and liver $(\boldsymbol{b}) 4 \mathrm{~d}$ after immune challenge in animals that were housed with a running wheel, a locked wheel, or no wheel for 6 weeks. Means \pm SEM are plotted. $\boldsymbol{a}$, Hippocampus. There was a significant main effect of physical activity (sedentary, locked, runners), $F_{(2,35)}=6.11, p>0.005$, a significant main effect of immune challenge (vehicle, $E$. coli), $F_{(1,35)}=15.21, p<0.0005$, and a significant physical activity $\times$ immune challenge interaction, $F_{(2,35)}=3.57, p<0.05$. Fisher's post hoc tests showed that all vehicle-treated rats exhibited comparable IL-1 $\beta$ protein levels, and did not differ across conditions. Sedentary E. coli-treated IL-1 $\beta$ levels $(n=5)$ were significantly higher compared with their vehicle controls $(p<0.01 ; n=6)$, E. coli-treated locked-wheel rats $(p<0.05 ; n=5)$, and $E$. coli-treated runners $(p<0.0005 ; n=8)$. Hippocampal IL- $1 \beta$ levels of locked-wheeled $E$. coli-treated rats were also significantly higher compared with their vehicle-treated controls $(p<0.005 ; n=6$ ) and $E$. coli-treated runners ( $p=0.05$ ). IL-1 $\beta$ levels in $E$. coli-treated runners were not different from their vehicle-treated controls $(p=0.73 ; n=$ 11). $\boldsymbol{b}$, Liver. There was a significant main effect of physical activity, $F_{(2,36)}=5.18, p<0.05$, a significant main effect of immune challenge, $F_{(1,36)}=41.98, p<0.0001$, and a significant physical activity $\times$ immune challenge interaction, $F_{(2,36)}=6.05, p<0.005$. Again, all vehicletreated rats exhibited comparable IL- $1 \beta$ protein levels, and did not differ across activity conditions. However, IL-1 $\beta$ levels in sedentary $E$. coli-treated rats $(n=7)$ were significantly higher compared with their vehicle controls $(p<0.0005 ; n=7)$, E. coli-treated locked wheel rats $(p<$ $0.05 ; n=4)$, and $E$. coli-treated runners $(p<0.05 ; n=8)$. Liver IL-1 $\beta$ levels of locked wheel E. coli-treated rats were also significantly higher compared with their vehicle-treated controls $(p<0.0001 ; n=5)$, but not different from E. coli-treated runners ( $p>0.05)$. Liver IL- $1 \beta$ levels in $E$. coli-treated runners were also significantly higher compared with their vehicle-treated controls $(p<0.001 ; n=11)$.

IL-6

Again, LPS increased expression of IL-6 in a dose-dependent manner. However, exercise only reduced the IL-6 mRNA increase at the highest LPS dose (Fig. $5 c$ ). Given that IL-6 induction lags behind TNF $\alpha$ and IL-1 $\beta$ (Givalois et al., 1994), the $2 \mathrm{~h}$ time point may not have been ideal for the observation of IL-6 increases.

Together, these data show that exercise robustly reduces the potentiated inflammatory response of aged hippocampal microglia to proinflammatory stimulation.

\section{Discussion}

It has been noted that cognitive declines during normal, healthy aging are often precipitous rather than gradual, and that these declines are frequently preceded by peripheral inflammatory challenges such as infection or injury (Holmes et al., 2003; Sparkman and Johnson, 2008; Barrientos et al., 2010). However, the
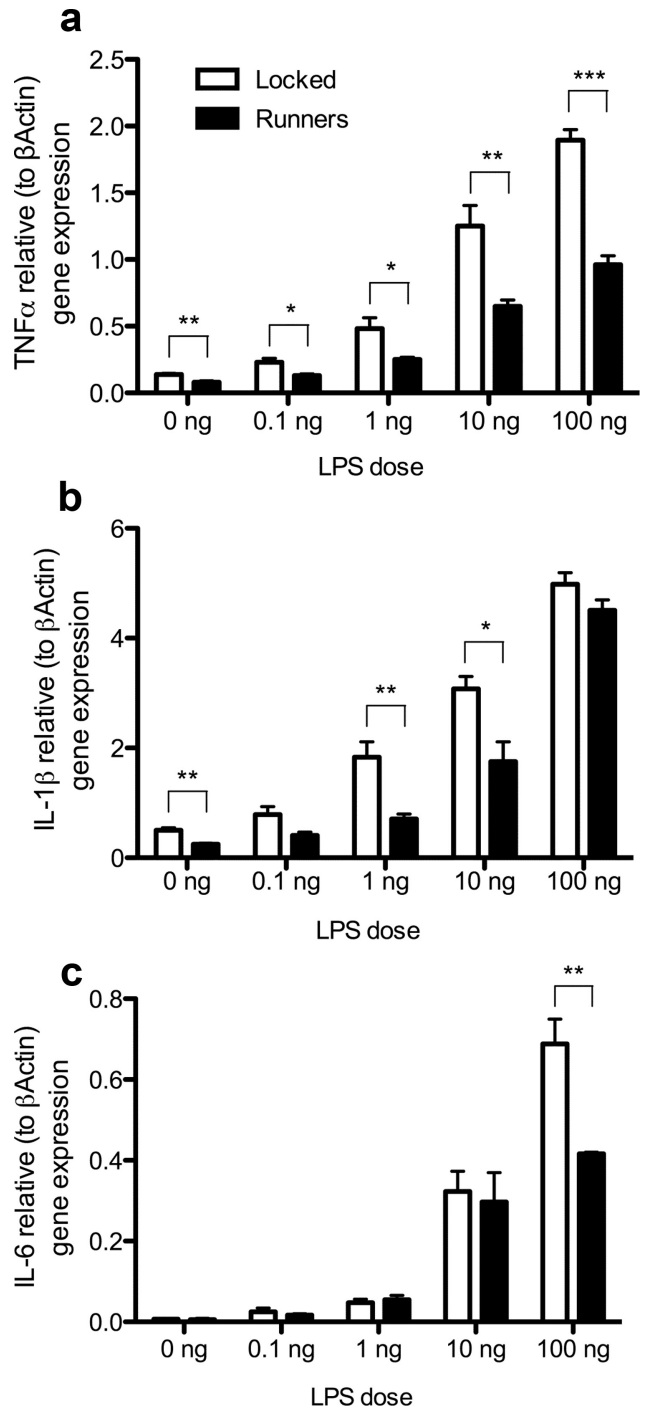

Figure 5. $\boldsymbol{a}-\boldsymbol{c}, \operatorname{TNF} \alpha(\boldsymbol{a}), \mathrm{IL}-1 \beta(\boldsymbol{b})$, and IL-6 (c) gene expression in isolated microglia $2 \mathrm{~h}$ following stimulation with increasing doses of LPS. Means \pm SEM are plotted; $n=4$ in each group. $\boldsymbol{a}, \operatorname{TNF} \alpha$. There was a significant main effect of physical activity (locked, runners), $F_{(1,24)}=$ $37.03, p<0.001$, a significant main effect of LPS dose $(0,0.1,1,10,100 \mathrm{ng}), F_{(4,24)}=206.29, p<$ 0.0001 , and a significant physical activity $\times$ LPS dose interaction, $F_{(4,24)}=22.45, p<0.0001$. At all doses of LPS, even the 0 ng dose, TNF gene expression was significantly higher in microglia of locked wheel rats than that of runners $(p<0.05) \cdot \boldsymbol{b}, \mathrm{IL}-1 \beta$. There was a significant main effect of physical activity, $F_{(1,24)}=17.23, p<0.01$, a significantmain effect of LPS dose, $F_{(4,24)}=224.78, p<0.0001$, and a significant physical activity $\times$ LPS dose interaction, $F_{(4,24)}=4.07, p<0.01$. IL- $1 \beta$ gene expression was significantly higher in microglia of locked wheel rats than that of runners at the $0 \mathrm{ng}$ $(p<0.002), 0.1 \mathrm{ng}(p=0.05), 1 \mathrm{ng}(p<0.01)$, and $10 \mathrm{ng}(p<0.05)$ doses, but not the $100 \mathrm{ng}$ dose $(p>0.05)$. c, L-6. There was no significant main effect of physical activity, $F_{(1,24)}=4.35, p>0.05$, a significant main effect of $L P S$ dose, $F_{(4,24)}=117.19, p<0.0001$, and a significant physical activity $\times$ LPS dose interaction, $F_{(4,24)}=7.35, p<0.0005$. IL-6 gene expression was significantly higher in microglia of locked wheel rats than that of runners only at the $100 \mathrm{ng}$ dose $(p<0.005)$.

mechanisms responsible for these declines are not understood. This led us to study aging rats (not senescent) that show unimpaired cognitive function on standard memory tasks before an infection, but exhibit prolonged impairments of long-term memory following infection (Barrientos et al., 2006). The duration of these impairments perfectly parallel the duration of IL- $1 \beta$ elevations in the hippocampus (Barrientos et al., 2009), and intracerebral administration of IL-1RA blocks these effects (Frank et al., 2010b). Given these previous findings, proinflammatory cytokines such as IL-1 $\beta$ clearly play a prominent role in the pro- 
cesses underlying these impairments. One likely possibility is that IL- $1 \beta$ alters downstream mediators important to memory formation. BDNF appears to be a strong candidate in this regard, as it has been repeatedly implicated as key for plasticity and the formation of memories (Tyler et al., 2002; Soule et al., 2006), and it is known to be downregulated by IL- $1 \beta$ (Barrientos et al., 2004). To further explore this issue, we have examined LTP in hippocampal slices of young and aged subjects that had received E. coli or vehicle $4 \mathrm{~d}$ earlier. E. coli abrogated only forms of LTP that are heavily dependent on BDNF, and only in aged rats (Chapman et al., 2010).

It is known that exercise can increase hippocampal BDNF expression (Cotman et al., 2007) as well as improve cognitive function in a number of situations (Woods et al., 2002; Greenwood et al., 2009; Erickson et al., 2011). Thus, we sought to determine whether it might prevent/reverse aging-induced susceptibility to memory impairments produced by peripheral immune challenge, and whether it would alter the age-related changes thought to mediate this impairment. Six weeks of access to a running wheel completely eliminated the memory impairment produced by $E$. coli in aged subjects. This effect was not produced by the provision of a locked running wheel, so activity in the wheel seems to be required. Remarkably, this effect occurred even though the aging subjects ran very little, only $\sim 700 \mathrm{~m}$ a week on average, nearly 50 times less than does a young rat. Nonetheless, the activity was enough to produce an $\sim 6 \%$ body weight reduction within 2 weeks of the intervention, and to maintain that reduction over the duration of the study ( $6-7$ weeks). Moreover, the finding that the susceptibility to memory impairment is present at 22.5 months suggests that exercise reversed age-related processes that had already developed.

As noted, BDNF induced after learning and maintained during the consolidation period is critical for long-term memory formation. In the present study, infection markedly blunted BDNF mRNA induction in the CA1 region of the hippocampus of sedentary and locked wheel rats. However, exercise potently prevented the blunting effects of infection on BDNF levels, as these levels were as high in infected runners as in vehicle-treated runners. There were no significant differences in BDNF expression between vehicle-treated sedentary and running rats, an effect which conflicts with what has been shown in young animals (Cotman et al., 2007). This may be due to the large discrepancy in the amounts of exercise achieved between aged and young rats, but this explanation is only speculative.

Increased hippocampal IL- $1 \beta$ is a likely cause of the BDNF reductions found here because (1) intrahippocampal administration of IL- $1 \beta$ reduces BDNF (Barrientos et al., 2004), (2) peripheral E. coli increases hippocampal IL-1 $\beta$ (Barrientos et al., 2009), and (3) intracerebral administration of IL-1RA blocks hippocampal BDNF reductions produced by E. coli (Cortese et al., 2011). Here, IL-1 $\beta$ in the hippocampus was indeed significantly elevated in both sedentary and locked wheel subjects following infection. Strikingly however, rats that exercised showed no such elevation. This could be the critical protection produced by running, given the likely role of hippocampal IL- $1 \beta$ in producing hippocampal-dependent memory impairments (Gemma and Bickford, 2007; Barrientos et al., 2010), BDNF reductions (Lapchak et al., 1993; Cortese et al., 2011) and impairments in associated processes such as LTP (Chapman et al., 2010; Lynch, 2010).

There are two different, but not mutually exclusive, global possibilities concerning why aging leads to exaggerated brain IL- $1 \beta$ increases following peripheral infection. It is possible that aging sensitizes the brain machinery that produces IL- $1 \beta$ upon receiving signals from the periphery, or aging could sensitize the peripheral machinery that signals the brain during infection. The latter possibility was examined previously, with the finding being that aging does not increase the proinflammatory cytokine response to E. coli in the periphery (serum or spleen) (Barrientos et al., 2009). However, liver changes were not examined in that study, and the liver now appears to be a key structure in leading to immune-to-brain signals (Campbell et al., 2007). Thus, liver IL- $1 \beta$ levels were examined here. While the ability to exercise blunted liver IL- $1 \beta$ increases compared with that of sedentary rats, those levels did not differ from infected rats that had locked wheels. Since these two groups did differ significantly on memory performance, these findings suggest that proinflammatory cytokine levels in the liver are not as critical as those in the hippocampus for producing, or preventing, hippocampal-dependent memory deficits.

Previous work has indicated that aging does sensitize the brain machinery that produces IL-1 $\beta$ (Perry, 2004; Frank et al., 2006, 2010a; Henry et al., 2009). This work has focused on microglia, since microglia are generally viewed as the major source of brain IL-1 $\beta$ (Van Dam et al., 1995). We thus examined isolated hippocampal microglia in the present studies. Microglia isolated from rats that were able to run for 6 weeks showed a markedly reduced proinflammatory response to LPS compared with microglia isolated from rats that had locked wheels. That is, running reduced the sensitivity of microglia to stimulation, and this may be at the core of the mechanism by which running protects the aging subjects. Several mechanisms have been suggested as to how aging might sensitize microglia. Microglia are maintained in a quiescent state by ligation of either the fractalkine (CX(3)CR1) or CD200 receptor (Hoek et al., 2000; Cardona et al., 2006), both expressed on the microglial cell surface. The ligands for these receptors (CX(3)CL1, CD200, respectively) are expressed on neurons, and so contact with neurons reduces microglial activity (Hoek et al., 2000). Aging has been shown to reduce both CX(3)CR1 (Wynne et al., 2010) and CD200 (Frank et al., 2006; Lyons et al., 2007). Thus, it is possible that running restores signaling in either or both of these pathways. Since LPS is a Toll-like receptor 4 (TLR4) ligand, it can also be noted that aging upregulates TLR expression in brain (Letiembre et al., 2007). Since there are also endogenous ligands that activate TLRs (Bianchi, 2007), it is also possible that exercise reduces microglial sensitivity by decreasing TLR expression. In any case, the present report is the first to show that running can reduce microglial sensitivity to stimulation.

In sum, the data strongly support the view that aging produces cognitive vulnerability to peripheral immune challenge because it sensitizes microglia, leading to exaggerated brain proinflammatory responses to the challenge, thereby interfering with BDNF expression in the period after learning. This hypothesis requires that any manipulation which blunts memory declines after infection also reduce microglial sensitivity, IL- $1 \beta$ increases, and BDNF decreases, and conversely. This is precisely the pattern of results presented here.

Exercise in humans has been shown to protect against declines in cognitive function with aging. For example, recent reports showed that aerobic exercise increased hippocampal volume and improved spatial memory (Erickson et al., 2011) and delayed the onset of cognitive impairments (Jedrziewski et al., 2010) in older individuals. It was concluded that exercise lowers the risk of dementia. It has been noted that dementia is often preceded by peripheral infection or other immune challenges (Holmes et al., 2003), and so the present data offer a possible mechanism for the protection afforded by physical activity. It should be noted that 
aging-associated cognitive declines may arise from various different insults targeting different brain regions and/or functions. Therefore, depending upon the etiology of the cognitive decline, physical exercise may exert its beneficial effects through very different mechanisms. For instance, in the TgCRND8 mouse model of Alzheimer's disease, exercise has been demonstrated to reduce amyloid load by improving energy metabolism in hippocampal neurons (Adlard et al., 2005; García-Mesa et al., 2011). In a model of stroke, however, exercise improved cerebral outcomes by increasing endothelial nitric oxide synthase, thereby increasing the density of perfused vasculature and cerebral blood flow (Gertz et al., 2006). In the present study, we conclude that even small to moderate amounts of physical activity protect against infectioninduced cognitive loss by normalizing microglial-driven neuroinflammatory responses to peripheral immune activation.

\section{References}

Adlard PA, Perreau VM, Pop V, Cotman CW (2005) Voluntary exercise decreases amyloid load in a transgenic model of Alzheimer's disease. J Neurosci 25:4217-4221.

Altschul SF, Madden TL, Schäffer AA, Zhang J, Zhang Z, Miller W, Lipman DJ (1997) Gapped BLAST and PSI-BLAST: a new generation of protein database search programs. Nucleic Acids Res 25:3389-3402.

Barrientos RM, Sprunger DB, Campeau S, Higgins EA, Watkins LR, Rudy JW, Maier SF (2003) Brain-derived neurotrophic factor mRNA downregulation produced by social isolation is blocked by intrahippocampal interleukin-1 receptor antagonist. Neuroscience 121:847-853.

Barrientos RM, Sprunger DB, Campeau S, Watkins LR, Rudy JW, Maier SF (2004) BDNF mRNA expression in rat hippocampus following contextual learning is blocked by intrahippocampal IL-1beta administration. J Neuroimmunol 155:119-126.

Barrientos RM, Higgins EA, Biedenkapp JC, Sprunger DB, Wright-Hardesty KJ, Watkins LR, Rudy JW, Maier SF (2006) Peripheral infection and aging interact to impair hippocampal memory consolidation. Neurobiol Aging 27:723-732.

Barrientos RM, Frank MG, Hein AM, Higgins EA, Watkins LR, Rudy JW, Maier SF (2009) Time course of hippocampal IL-1 beta and memory consolidation impairments in aging rats following peripheral infection. Brain Behav Immun 23:46-54.

Barrientos RM, Frank MG, Watkins LR, Maier SF (2010) Memory impairments in healthy aging: role of aging-induced microglial sensitization. Aging Dis 1:212-231.

Bianchi ME (2007) DAMPs, PAMPs and alarmins: all we need to know about danger. J Leukoc Biol 81:1-5.

Campbell SJ, Jiang Y, Davis AE, Farrands R, Holbrook J, Leppert D, Anthony DC (2007) Immunomodulatory effects of etanercept in a model of brain injury act through attenuation of the acute-phase response. J Neurochem 103:2245-2255.

Cardona AE, Pioro EP, Sasse ME, Kostenko V, Cardona SM, Dijkstra IM, Huang D, Kidd G, Dombrowski S, Dutta R, Lee JC, Cook DN, Jung S, Lira SA, Littman DR, Ransohoff RM (2006) Control of microglial neurotoxicity by the fractalkine receptor. Nat Neurosci 9:917-924.

Chapman TR, Barrientos RM, Ahrendsen JT, Maier SF, Patterson SL (2010) Synaptic correlates of increased cognitive vulnerability with aging: peripheral immune challenge and aging interact to disrupt theta-burst latephase long-term potentiation in hippocampal area CA1. J Neurosci 30:7598-7603.

Cortese GP, Barrientos RM, Maier SF, Patterson SL (2011) Aging and a peripheral immune challenge interact to reduce mature brain-derived neurotrophic factor and activation of TrkB, PLC $\{$ gamma $\} 1$, and ERK in hippocampal synaptoneurosomes. J Neurosci 31:4274-4279.

Cotman CW, Berchtold NC, Christie LA (2007) Exercise builds brain health: key roles of growth factor cascades and inflammation. Trends Neurosci 30:464-472.

Erickson KI, Voss MW, Prakash RS, Basak C, Szabo A, Chaddock L, Kim JS, Heo S, Alves H, White SM, Wojcicki TR, Mailey E, Vieira VJ, Martin SA, Pence BD, Woods JA, McAuley E, Kramer AF (2011) Exercise training increases size of hippocampus and improves memory. Proc Natl Acad Sci U S A 108:3017-3022.
Fanselow MS (1990) Factors governing one trial contextual conditioning. Anim Learn Behav 18:264-270.

Fanselow MS, Lester LS (1988) A functional behavioristic approach to aversively motivated behavior: predatory imminence as a determinant of the topography of defensive behavior. In: Evolution and learning (Bolles RC, Beecher MD, eds), pp 185-211. Hillside, NJ: Erlbaum.

Frank MG, Barrientos RM, Biedenkapp JC, Rudy JW, Watkins LR, Maier SF (2006) mRNA up-regulation of MHC II and pivotal pro-inflammatory genes in normal brain aging. Neurobiol Aging 27:717-722.

Frank MG, Barrientos RM, Watkins LR, Maier SF (2010a) Aging sensitizes rapidly isolated hippocampal microglia to LPS ex vivo. J Neuroimmunol 226:181-184.

Frank MG, Barrientos RM, Hein AM, Biedenkapp JC, Watkins LR, Maier SF (2010b) IL-1RA blocks E. coli-induced suppression of Arc and long-term memory in aged F344×BN F1 rats. Brain Behav Immun 24:254-262.

García-Mesa Y, López-Ramos JC, Giménez-Llort L, Revilla S, Guerra R, Gruart A, Laferla FM, Cristòfol R, Delgado-García JM, Sanfeliu C (2011) Physical Exercise Protects Against Alzheimer's Disease in 3xTg-AD Mice. J Alzheimers Dis 24:421-454.

Gemma C, Bickford PC (2007) Interleukin-1beta and caspase-1: players in the regulation of age-related cognitive dysfunction. Rev Neurosci $18: 137-148$

Gertz K, Priller J, Kronenberg G, Fink KB, Winter B, Schröck H, Ji S, Milosevic M, Harms C, Böhm M, Dirnagl U, Laufs U, Endres M (2006) Physical activity improves long-term stroke outcome via endothelial nitric oxide synthase-dependent augmentation of neovascularization and cerebral blood flow. Circ Res 99:1132-1140.

Givalois L, Dornand J, Mekaouche M, Solier MD, Bristow AF, Ixart G, Siaud P, Assenmacher I, Barbanel G (1994) Temporal cascade of plasma level surges in ACTH, corticosterone, and cytokines in endotoxin-challenged rats. Am J Physiol 267:R164-R170.

Godbout JP, Chen J, Abraham J, Richwine AF, Berg BM, Kelley KW, Johnson RW (2005) Exaggerated neuroinflammation and sickness behavior in aged mice following activation of the peripheral innate immune system. FASEB J 19:1329-1331.

Greenwood BN, Strong PV, Foley TE, Fleshner M (2009) A behavioral analysis of the impact of voluntary physical activity on hippocampus-dependent contextual conditioning. Hippocampus 19:988-1001.

Hall J, Thomas KL, Everitt BJ (2000) Rapid and selective induction of BDNF expression in the hippocampus during contextual learning. Nat Neurosci 3:533-535.

Henry CJ, Huang Y, Wynne AM, Godbout JP (2009) Peripheral lipopolysaccharide (LPS) challenge promotes microglial hyperactivity in aged mice that is associated with exaggerated induction of both proinflammatory IL-1beta and anti-inflammatory IL-10 cytokines. Brain Behav Immun 23:309-317.

Hoek RM, Ruuls SR, Murphy CA, Wright GJ, Goddard R, Zurawski SM, Blom B, Homola ME, Streit WJ, Brown MH, Barclay AN, Sedgwick JD (2000) Down-regulation of the macrophage lineage through interaction with OX2 (CD200). Science 290:1768-1771.

Holmes C, El-Okl M, Williams AL, Cunningham C, Wilcockson D, Perry VH (2003) Systemic infection, interleukin 1beta, and cognitive decline in Alzheimer's disease. J Neurol Neurosurg Psychiatry 74:788-789.

Jedrziewski MK, Ewbank DC, Wang H, Trojanowski JQ (2010) Exercise and cognition: results from the National Long Term Care Survey. Alzheimers Dement 6:448-455.

Lapchak PA, Araujo DM, Hefti F (1993) Systemic interleukin-1 beta decreases brain-derived neurotrophic factor messenger RNA expression in the rat hippocampal formation. Neuroscience 53:297-301.

Letiembre M, Hao W, Liu Y, Walter S, Mihaljevic I, Rivest S, Hartmann T, Fassbender K (2007) Innate immune receptor expression in normal brain aging. Neuroscience 146:248-254.

Lynch MA (2010) Age-related neuroinflammatory changes negatively impact on neuronal function. Front Aging Neurosci 1:6.

Lyons A, Downer EJ, Crotty S, Nolan YM, Mills KH, Lynch MA (2007) CD200 ligand receptor interaction modulates microglial activation in vivo and in vitro: a role for IL-4. J Neurosci 27:8309-8313.

Maier SF, Watkins LR (1998) Cytokines for psychologists: implications of bidirectional immune-to-brain communication for understanding behavior, mood, and cognition. Psychol Rev 105:83-107. 
Nickerson M, Elphick GF, Campisi J, Greenwood BN, Fleshner M (2005) Physical activity alters the brain Hsp72 and IL-1beta responses to peripheral E. coli challenge. Am J Physiol Regul Integr Comp Physiol 289:R1665-R1674.

Perry VH (2004) The influence of systemic inflammation on inflammation in the brain: implications for chronic neurodegenerative disease. Brain Behav Immun 18:407-413.

Rudy JW, Barrientos RM, O’Reilly RC (2002) Hippocampal formation supports conditioning to memory of a context. Behav Neurosci 116:530-538.

Soulé J, Messaoudi E, Bramham CR (2006) Brain-derived neurotrophic factor and control of synaptic consolidation in the adult brain. Biochem Soc Trans 34:600-604.

Sparkman NL, Johnson RW (2008) Neuroinflammation associated with aging sensitizes the brain to the effects of infection or stress. Neuroimmunomodulation 15:323-330.

Tyler WJ, Alonso M, Bramham CR, Pozzo-Miller LD (2002) From ac- quisition to consolidation: on the role of brain-derived neurotrophic factor signaling in hippocampal-dependent learning. Learn Mem 9: 224-237.

Van Dam AM, Bauer J, Tilders FJ, Berkenbosch F (1995) Endotoxininduced appearance of immunoreactive interleukin-1 beta in ramified microglia in rat brain: a light and electron microscopic study. Neuroscience 65:815-826.

Vaynman S, Ying Z, Gomez-Pinilla F (2004) Hippocampal BDNF mediates the efficacy of exercise on synaptic plasticity and cognition. Eur J Neurosci 20:2580-2590.

Woods JA, Lowder TW, Keylock KT (2002) Can exercise training improve immune function in the aged? Ann N Y Acad Sci 959:117-127.

Wynne AM, Henry CJ, Huang Y, Cleland A, Godbout JP (2010) Protracted downregulation of CX(3)CR1 on microglia of aged mice after lipopolysaccharide challenge. Brain Behav Immun 24:1190-1201. 